\title{
Rotina de atendimentos fisioterapêuticos baseado em níveis de prioridade em uma unidade de neurocirurgia de um hospital público
}

\author{
Physiotherapeutic Based Care Routine on Priority Levels in \\ a Neurosurgery Unit of a Public Hospital
}

\section{Rutina de Fisioterapia Basada em Niveles de Prioridad em uma Unidad de Neurocirurgía de um Hospital Público}

\author{
Ana Cristina Ferreira dos Reis Almeida ${ }^{1}$, Carolinne Santos de \\ Oliveira ${ }^{2}$, Thais Souza Lacerda ${ }^{2}$, Maria Clara Henrique de Lima ${ }^{3}$, \\ Luciana Leite Melo e Silva ${ }^{2}$, Hudson Azevedo Pinheiro ${ }^{1,2,3}$
}

\begin{abstract}
1.Fisioterapeuta, Secretaria Estadual de Saúde do Distrito Federal (SESDF), Brasília-DF, Brasil.
2.Curso de Fisioterapia, Centro Universitário EuroAmericano de Brasília (UniEuro), Brasília-DF, Brasil.

3.Programa de Residências Multiprofissional em Saúde do Adulto e do Idoso, Escola Superior em
\end{abstract} Ciências da Saúde (ESCS/SESDF). Brasília-DF, Brasil.

\section{Resumo}

Objetivo. Verificar os efeitos de uma rotina de atendimentos fisioterapêuticos em uma Unidade de Neurocirurgia de um hospital público do Distrito Federal na mobilidade e tempo de internação dos pacientes e níveis de prioridade. Métodos. Trata-se de um estudo observacional descritivo de registros de pacientes internados na Unidade de Neurocirurgia (UNC), onde os dados foram coletados no período de 01 de outubro a 31 de dezembro de 2018, por meio do prontuário eletrônico no sistema de informatização Trackcare $®$, foram incluídos nesse estudo todos os internados da UNC durante o período de coleta, excluindo crianças, jovens, internação social ou judicial, e causas que não são da competência da UNC. Resultados. Essa pesquisa obteve um total de 124 pacientes, sendo verificado o perfil desses pacientes, bem como o nível de prioridade de fisioterapia da UNC, a fim de analisar a real necessidade da realização do atendimento fisioterapêutico, portanto foi demonstrada diferença estatisticamente significativa entre os grupos, comprovando que a classificação foi efetiva para a melhora da mobilidade desses pacientes. Conclusão. A escala IMS na admissão determinou os níveis de prioridade de atendimento fisioterapêutico na unidade de neurocirurgia, essa prioridade resultou em melhoras da mobilidade dos pacientes internados no momento da alta.

Unitermos. Unidades Hospitalares; Serviço hospitalar de fisioterapia; Procedimentos Neurocirúrgicos; Reabilitação Neurológica; Limitação de Mobilidade

\footnotetext{
Abstract

Objective. Verify the effects of a physiotherapeutic assistance routine in one Neurosurgery Unit of a public hospital in Federal District on mobility and hospital stay of patients and priority. Method. It is a descriptive observational study about registry of impatiens in Neurosurgery Unit (UNC) where the data were collected in the period of October 1 to December 31 of 2018 , by means of the electronic medical record in the computerization system Trackcare $\Re$, were included in this study all the internees of the UNC during the collection period, being excluded children, adolescents, social or judicial admission and causes that are not of the UNC domains. Results. This study obtained a total of 124 patients being verified the profile of these patients, as well as the physiotherapy priority level of UNC, in order to analyze the real need to perform physical therapy care therefore was shown a statistically significant difference between the groups proving that the classification was effective to improve the mobility of these patients. Conclusion. The IMS scale at the
} 
admission determined the priority levels of physiotherapeutic care in the neurosurgery unit, and this priority resulted in improvements in the mobility of hospitalized patients at the time of discharge.

Keywords. Hospital Units; Physiotherapy Hospital Service; Neurosurgical Procedures; Neurological Rehabilitation; Mobility Limitation

\section{Resumen}

Objetivo. Verificar los efectos de una atención fisioterapéutica de rutina en una Unidad de Neurocirugía de un hospital público en el Distrito Federal sobre la movilidad del paciente y la duración de la estadía y los niveles de prioridad. Métodos. Este es un estudio descriptivo observacional de registros de pacientes ingresados en la Unidad de Neurocirugía (UNC), donde se recopilaron datos del 1 de octubre al 31 de diciembre de 2018, a través del registro médico electrónico en el sistema informático. Trackcare ${ }^{\circledR}$ incluyó en este estudio a todos los internos de UNC durante el período de recolección, excluyendo niños, jóvenes, internamiento social o judicial, y causas que no están dentro de la competencia de UNC. Resultados. Este estudio obtuvo un total de 124 pacientes y se verificó el perfil de estos pacientes, así como el nivel de prioridad de fisioterapia de la UNC, para analizar la necesidad real de atención fisioterapéutica, por lo que se demostró una diferencia estadísticamente significativa entre demostrando que la clasificación fue efectiva para mejorar la movilidad de estos pacientes. Conclusión. la escala IMS al ingreso determinó los niveles de prioridad de la atención de fisioterapia en la unidad de neurocirugía, esta prioridad resultó en una mejor movilidad de los pacientes hospitalizados al alta.

Palabras clave. Unidades hospitalarias; servicio de fisioterapia en el hospital; procedimientos neuroquirúrgicos; Rehabilitación neurológica; Limitación de movimiento

Trabalho realizado na Escola Superior em Ciências da Saúde, Brasília-DF, Brasil.

Conflito de interesse: não Recebido em: 16/08/2019 Aceito em: 20/12/2019

Endereço para correspondência: Hudson A Pinheiro. R. 36 norte It 05 bl A apto 401, Águas Claras. Brasília-DF, Brasil. CEP 71919-180. Email: hudsonap@gmail.com

\section{INTRODUÇÃO}

A unidade neurocirúrgica (UNC) é um ambiente hospitalar que detém requisitos técnicos e equipamentos especializados para 0 tratamento dos pacientes neurológicos de alta complexidade, exercendo assim de forma técnica programas e protocolos estabelecidos pelo sistema único de saúde (SUS) para gerar, com eficácia e eficiência, o atendimento dos doentes neurológicos, por meio de uma equipe multidisciplinar, para garantir a recuperação do quadro clínico desses pacientes ${ }^{1,2}$. 
A fisioterapia se faz presente buscando a funcionalidade através do movimento humano, favorecendo a recuperação das plegias/ou paresias, reduzindo os efeitos da imobilidade prolongada, como as complicações respiratórias e a redução da massa muscular, podendo inclusive contribuir para minimizar alterações emocionais, como ansiedade e depressão ${ }^{3-6}$.

No contexto da saúde pública em Brasília-DF, a fisioterapia atua nas unidades intensivas, nos ambulatórios de reabilitação física e funcional, nas enfermarias e no atendimento domiciliar e com base na atual resoluçãoCOFFITO n444/2014, que não especifica claramente, a quantidade exata de fisioterapeutas nas unidades de enfermaria, apenas especificando que esses profissionais com carga horária de seis horas deverão atender entre oito a 10 pacientes, desse modo é necessário à realização de triagens com critérios de inclusão, exclusão e alta para avaliar a real prioridade de atendimento de cada paciente ${ }^{7}$.

Conforme as diretrizes apresentadas pelo Ministério da Saúde, para o programa Humaniza SUS Ministério da Saúde (2008), os hospitais podem desenvolver protocolos validados para avaliar o nível de prioridade de atendimento. $\mathrm{Na}$ fisioterapia, da Unidade de Neurocirurgia (UNC) do Instituto Hospital de Base do Distrito Federal, adotou-se um protocolo que foi definido por níveis de prioridade, baseado no grau de restrição da mobilidade ativa, entretanto faz-se necessário verificar o impacto que esta rotina pode gerar no fluxo de atendimento de pacientes da $\mathrm{UNC}^{8-10}$. 
Portanto esse estudo tem como objetivo verificar o impacto de uma rotina de atendimentos fisioterapêuticos em uma Unidade de Neurocirurgia de um hospital público do Distrito Federal baseado em níveis de prioridade.

\section{MÉTODO}

\section{Amostra}

Trata-se de um estudo observacional descritivo retrospectivo de registros de pacientes internados na UNC do Instituto Hospital de Base do Distrito Federal (SUS) no período de 01 de outubro a 31 de dezembro de 2018. Esta pesquisa tem a aprovação do Comitê de Ética em pesquisa do Instituto Hospital de Base, Brasília, DF, Brasil, com Número do Parecer: 2.924.372.

\section{Procedimento}

Os dados do perfil e condições clínicas foram coletados das evoluções registradas continuadamente por todos os profissionais de saúde do setor, por intermédio de prontuário eletrônico no sistema de informatização TRACKCARE $®$, disponível apenas intranet no próprio hospital.

Os dados coletados foram idade, sexo, tempo de internação hospitalar, tempo de internação na UNC, CID, procedência, destino no momento da alta da Unidade, situação de pré ou pós-operatório, se faz uso de oxigenoterapia, se tem via aérea artificial, pontuação na 
escala ICU Mobility Scale (IMS) na admissão e no momento da alta, além da classificação no nível de prioridade de atendimento fisioterapêutico.

A IMS é uma escala que é comumente utilizada nas UTI para a verificação da mobilidade ativa dos pacientes internados, visando auxiliar a equipe multidisciplinar na classificação e na evolução neuromotora durante o período de internação, analisando fatores que vão desde o controle de tronco a marcha com e sem auxílio, por meio de 11 itens que pontuam de 0 a 10 pontos $^{9}$.

Foram incluídos todos os pacientes que estavam internados na UNC durante o período de coleta, excluindo as crianças, jovens, internação social ou judicial, e causas que não são da competência da UNC, por exemplo, clínica médica.

A IMS é um instrumento que foi elaborado a princípio para mensurar o grau de mobilidade de pacientes internados na unidade de terapia intensiva (UTI), tendo em vista identificar o risco de complicações geradas pela baixa mobilidade/movimentação no leito; ela detém uma pontuação variando entre0 e 10, em um único domínio, sendo que a pontuação zero expressa uma baixa mobilidade, que neste caso é interpretada como o paciente que realiza apenas exercícios passivos no leito, e a pontuação 10 expõe uma alta mobilidade, deambulação independente e sem auxílio ${ }^{11}$.

De acordo com as diretrizes propostas pelo Ministério da Saúde no programa Humaniza SUS Ministério da Saúde 
(2008), que determina que cada ambiente hospitalar pode desenvolver seu próprio protocolo de classificação de prioridade, conforme as características regionais da população e sua capacidade de atendimento, em 2003, foi implementado pelos fisioterapeutas da UNC do presente hospital um protocolo que classifica os pacientes por nível de prioridade de atendimento da fisioterapia em três categorias distintas definidas pelo IMS: nível 1 atendimento uma vez ao dia, cinco vezes por semana mais orientações; nível 2 - atendimento uma vez ao dia, três vezes por semana mais orientações por meio de cartilhas explicativas desenvolvidas na própria UNC; e nível três atividades orientadas por meio de cartilhas e exercícios em grupo supervisionados pelos fisioterapeutas duas vezes por semana.

\section{Análise Estatística}

As informações obtidas foram armazenadas na planilha Microsoft Office Excel 2010, e posteriormente transcrita para o software SPSS versão 20, e optou-se para descrição dos dados numéricos média e desvio padrão e os dados categóricos, proporções.

Para a comparação os escores da escala IMS e os níveis de prioridades, utilizou-se o teste paramétrico de $\mathrm{t}$ Student pareado, com nível de significância 95\%. 


\section{RESULTADOS}

Foram identificados 143 pacientes internados nessa unidade na UNC, sendo que 10 destes prontuários eram de crianças ou adolescentes, três eram internação social e cinco por causas que não são da competência da UNC, logo foram excluídos da pesquisa, participando, portanto, 124 pacientes. As características destes usuários, referentes à internação, estão descritas na Tabela 1.

A Tabela 2 apresenta os critérios clínicos dos pacientes admitidos na Unidade de neurocirurgia durante a realização do estudo.

Para verificar se o nível de prioridade de fisioterapia tem efeito na rotina da UNC optou-se por verificar os escores da escala IMS na admissão e alta, notou-se diferenças estatisticamente significativas nos grupos que realizaram fisioterapia (N1 e N2), conforme demonstra a Figura 1.

Tabela 1. Características dos usuários admitidos na Unidade de Neurocirurgia entre outubro

\begin{tabular}{ll}
\hline Características da internação & Média (DP) \\
\hline Idade (anos) & $46,7 \pm 16,7$ \\
\hline Tempo de internação hospitalar (dias) & $44,2 \pm 25,4$ \\
\hline Tempo de internação na UNC (dias) & $26,3 \pm 18,4$ \\
\hline IMS na admissão UNC & $5,5 \pm 4,6$ \\
\hline IMS na alta UNC & $5,9 \pm 4,2$ \\
\hline
\end{tabular}

$\mathrm{UNC}=$ Unidade de neurocirurgia; IMS= ICU mobility scale. 
Tabela 2. Sobre o perfil dos pacientes admitidos na UNC.

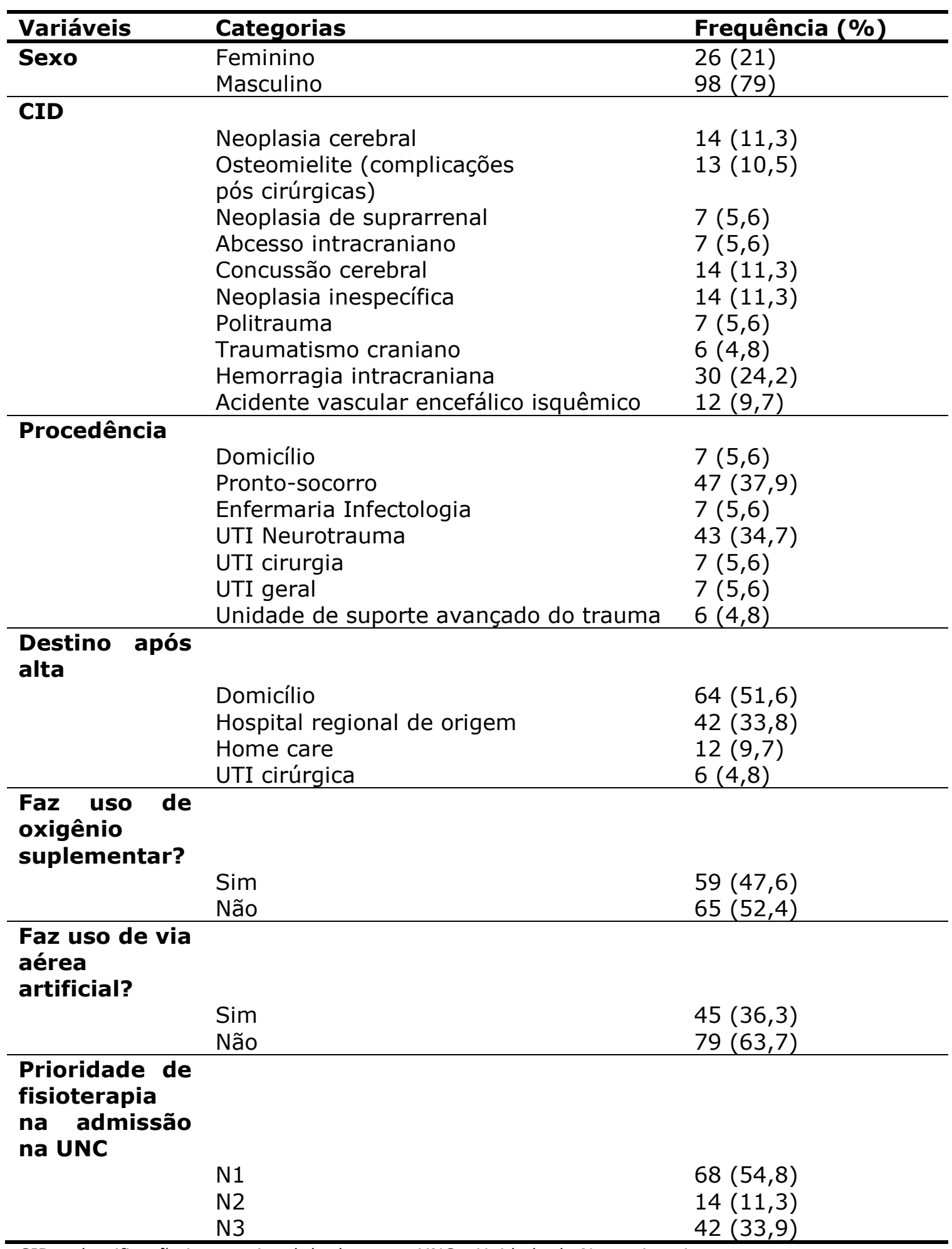

$\mathrm{CID}=$ classificação internacional de doenças; UNC = Unidade de Neurocirurgia. 
Figura 1. Comparação de escores de IMS na admissão e alta da UNC em relação à prioridade de atendimentos fisioterapêuticos.

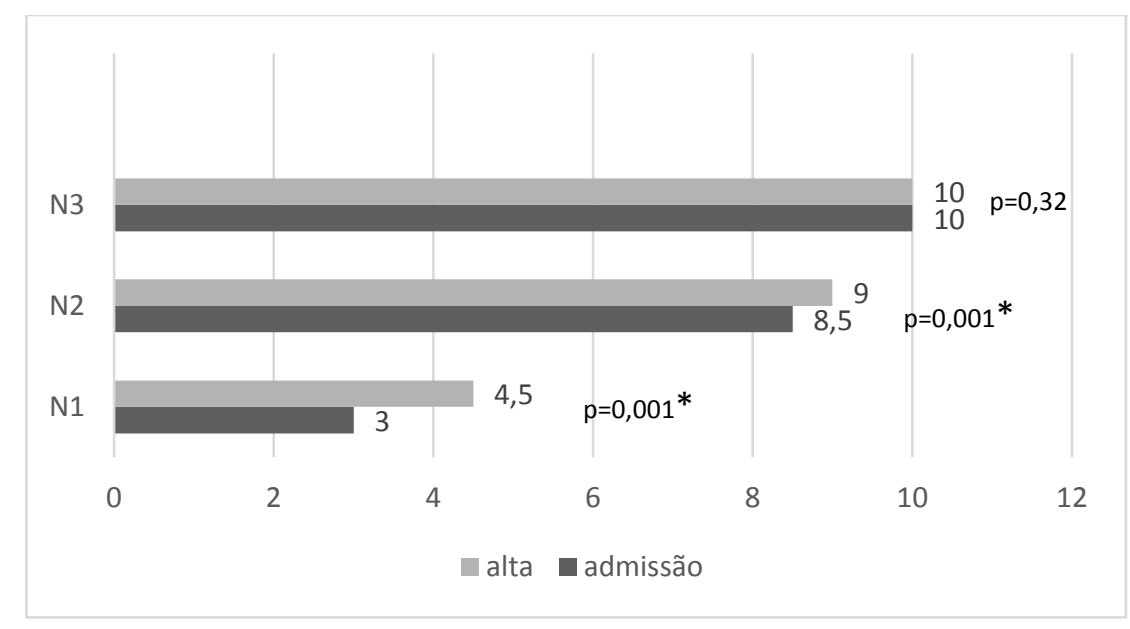

\section{DISCUSSÃO}

Analisando o perfil dos pacientes internados na UCN do Instituto Hospital de Base percebe-se que é constituído basicamente por adultos jovens com maior prevalência do sexo masculino, com 79\%, passando por períodos longos de internação com média de 45 dias e com desvio padrão de 25,4 .

Demonstra ser efetivo para reduzir o tempo de permanência e os custos hospitalares através da reabilitação precoce, onde é iniciado na unidade intensiva e com seu seguimento nas enfermarias, independentemente da idade, sexo, diagnóstico ou quantidade de dias internados na unidade intensiva, obteve um número significativo na alta hospitalar, quando comparado aos que não tiveram esse tipo de tratamento ${ }^{12}$.

No presente estudo observou-se que na classificação internacional de doenças (CID), houve uma maior prevalência da hemorragia intracraniana com 30 pacientes 
e com frequência de 24,2\%. Nessa amostra apresentou também o AVC isquêmico com 12 indivíduos e 9,7\%. Em contrapartida, a incidência do AVC hemorrágico entre 15 a $25 \%$ da população e os isquêmicos sendo os mais frequentes com 75 a $85 \%{ }^{13}$.

A incidência das neoplasias caracterizada como desconhecidas ou incertas do encéfalo e as neoplasias do sistema nervoso central, as duas com 14 pacientes cada, com 14,4\% frequentes. Aproximadamente 21.810 habitantes nos Estados Unidos com o diagnóstico de tumor cerebral maligno, onde 11.780 eram homens e 10.030 mulheres ${ }^{14}$.

Esse trabalho alcançou uma maior procedência de pacientes internados no pronto-socorro com frequência de $37,9 \%$ e nas UTI Neurotrauma de 34,7\%. Pode ser verificado também nesse estudo o destino desses pacientes após alta, onde 64 desses pacientes foram para seus domicílios e 42 direcionados para o hospital regional de origem e os restantes desses sendo encaminhados para home care e seis pacientes para UTI cirúrgica.

$\mathrm{Na}$ unidade de neurocirurgia 59 pacientes internados fizeram o uso da oxigenoterapia, e 54,2\% dessa população não fez uso do oxigênio suplementar. Em relação aos pacientes com via aérea artificial obteve um total de 36,2\% com traqueostomia e 63,7\% não precisaram fazer esse tipo de procedimento. Essas variáveis são importantes para analisar necessidade do atendimento fisioterapêutico. 
A prioridade de fisioterapia na UNC no momento da admissão obteve na classificação de nível 1 um total de $54,8 \%$ pacientes que precisavam realizar fisioterapia e $33,9 \%$ desses indivíduos eram do nível 3 e recebiam orientações e observações dos fisioterapeutas. Desse modo é possível observar que muitos desses pacientes precisaram realizar a fisioterapia nessa unidade.

A reabilitação neurológica de pacientes internados apresentaram resultados relevantes nas capacidades cognitivas, incluindo as habilidades de aprendizado, habilidades espaciais, resolutividade dos problemas, memória, velocidade de processamento e nas funções físicas e globais ${ }^{9}$. Devido à neuroplasticidade onde um ambiente estimulante provoca alterações moleculares e nas células perceptivas do cérebro; essa reabilitação também proporciona a interação desses pacientes na socialização, confiança e autoestima para o enfrentamento da sua condição neurológica ${ }^{15,16}$.

Nesse estudo a escala IMS determinou no momento da admissão os níveis de prioridade de atendimento fisioterapêutico na unidade de neurocirurgia. Essa escala é utilizada no primeiro momento para identificar a restrição motora, e a partir disso elencar a prioridade de atendimentos fisioterapêutico para reduzir os efeitos da reabilitação intra-hospitalar. Sendo esse instrumento usado por fisioterapeutas, no qual vem demonstrando sua importância na execução e promoção da reabilitação precoce $^{17}$. 
Foi observado nessa pesquisa melhorias na mobilidade quando comparado com os níveis de prioridade do grupo um e dois, que usufruíram de um maior contado com a fisioterapia e alcançaram uma diferença no escore de admissão da escala IMS quando comparados o momento da admissão com o da alta, ficando demonstrado que a realização da fisioterapia possui um efeito positivo dentro da UNC, contribuindo para o restauro da condição de saúde desses pacientes.

Como limitações, podemos mencionar a escassez de estudos sobre a rotina dos fisioterapeutas no contexto das enfermarias e das unidades de neurocirurgias, visto que a maioria das pesquisas é designada as emergências e as unidades de terapia intensiva (UTI). Do mesmo modo que não existe uma legislação que dê um respaldo sobre a quantidade exata de fisioterapeutas por leito nas enfermarias conforme ocorre na UTI, por exemplo. Desse modo sugerimos novas pesquisas sobre o contexto do fisioterapeuta nas enfermarias e nas unidades de neurocirurgia, bem como se há necessidade de criação de uma legislação que forneça um amparo para esses profissionais.

\section{CONCLUSÃO}

A escala IMS na admissão norteou níveis de prioridade de atendimento fisioterapêutico na unidade de 
neurocirurgia, e essa prioridade resultou em melhoras da mobilidade dos pacientes internados no momento da alta, conforme se observou ao longo desta pesquisa.

\section{REFERÊNCIAS}

1.França EÉT de, Ferrari F, Fernandes $P$, Cavalcanti $R$, Duarte A, Martinez BP, et al. Fisioterapia em pacientes críticos adultos: recomendações do Departamento de Fisioterapia da Associação de Medicina Intensiva Brasileira. Rev Bras Ter Intensiva 2010;24:6-22. http://dx.doi.org/10.1590/S0103-507X2012000100003

2.BRASIL. Resolução $n^{\circ}$ 444/14, de 26 de abril de 2014. Diário Oficial da União, Brasília, 26/04/2014, secção 1, p.104. https://www.coffito.gov.br/nsite/?p=3208

3. Hess D, Nishiwaki J, Liberatori MF, Eichinger FLF. Modalidades de Tratamento da Espasticidade: Uma Revisão da Literatura. Cad Edu Saúde e Fis 2017;4:46-56.

http://revista.redeunida.org.br/ojs/index.php/cadernos-educacaosaude-fisioter/article/view/870/pdf 36

4.Valente SCF, Paula EB, Abranches M, Costa V, Borges H, Chamlian $\mathrm{TR}$, et al. Resultados da fisioterapia hospitalar na função do membro superior comprometido após acidente vascular encefálico. Rev Neurocienc 2006;14:122-6.

http://revistaneurociencias.com.br/edicoes/2006/RN\%2014\%2003/P ages\%20from\%20RN\%2014\%2003.pdf

5.Azevedo PMD da S, Gomes BP. Efeitos da mobilização precoce na reabilitação funcional em doentes críticos: uma revisão sistemática. Rev Enf Ref 2015;5:129-38. http://dx.doi.org/10.12707/RIV14035 6.Pereira HCB, Duarte PHM, do Monte Mélo T, da Silva RMC, dos Santos WV, de Souza Barbosa $D$, et al. Intervenção fisioterapêutica na Síndrome da Imobilidade em pessoas idosas: revisão sistematizada. Arch Heal Investig 2017;6:505-8.

http://dx.doi.org/10.21270/archi.v6i11.2242

7.Gerência de Saúde Funcional. Conduta Fisioterapêuticas nas Enfermarias da Secretaria de Estado de Saúde do DF. Portaria SESDF No 29 de $1^{\circ}$ de Março de 2016 , publicada no DODF No 42 de 3 de Março de 2016, p.1-41.

http://www.saude.df.gov.br/wp-conteudo/uploads/2018/04/3.Conduta Fisioterapeutica nas Enfermarias da SES-DF.pdf 8.Ministério da Saúde. Envelhecimento e saúde da pessoa idosa. Cadernos de Atenção Básica nº19. 2006, 192p. http://bvsms.saude.gov.br/bvs/publicacoes/evelhecimento saude pe ssoa idosa.pdf

9.Khan F, Amatya B, Elmalik A, Lowe $\mathrm{M}, \mathrm{Ng} \mathrm{L}$, Reid I, et al. An enriched environmental programme during inpatient neuro- 
rehabilitation: A randomized controlled trial. J Rehabil Med 2016;48:417-25. http://dx.doi.org/10.2340/16501977-2081

10.Ferreira J, Silva JCA, Cavalcante TB, Campelo GO. Atuação do fisioterapeuta em enfermaria hospitalar no Brasil. Fisioter Bras 2017;18. http://dx.doi.org/10.33233/fb.v18i6.1055

11.Kawaguchi YMF, Nawa RK, Figueiredo TB, Martins L, Pires-Neto RC. Perme Intensive Care Unit Mobility Score e ICU Mobility Scale: tradução e adaptação cultural para a língua portuguesa falada no Brasil. J Bras Pneumol 2016;42:429-31.

http://dx.doi.org/10.1590/s1806-37562015000000301

12.Gruther W, Pieber K, Steiner I, Hein C, Hiesmayr JM, PaternostroSluga T. Can early rehabilitation on the general ward after an intensive care unit stay reduce hospital length of stay in survivors of critical illness?: a randomized controlled trial. Am J Phys Med Rehabil 2017;96:607-15.

http://dx.doi.org/10.1097/PHM.0000000000000718

13.Silva IFG da, Neves CF da S, Vilela ACG, Bastos LMD, Henriqueset MILS. Viver e cuidar após o acidente vascular cerebral. Rev Enferm Ref 2016;8:103-11. http://dx.doi.org/10.12707/RIV15047

14.Bondy ML, Scheurer ME, Malmer B, Barnholtz-Sloan JS, Davis FG, Il'yasova D, et al. Brain tumor epidemiology: consensus from the Brain Tumor Epidemiology Consortium. Cancer 2008;113:1953-68. http://dx.doi.org/10.1002/cncr.23741.

15.Levin MF, Kleim JA, Wolf SL. What do motor "recovery" and "compensation" mean in patients following stroke? Neurorehabil Neural Repair 2009;23:313-9.

http://dx.doi.org/10.1177/1545968308328727

16.Langhorne P, Coupar F, Pollock A. Motor recovery after stroke : a systematic review. Lancet Neurol 2009;8:741-54.

http://dx.doi.org/10.1016/S1474-4422(09)70150-4

17. Hodgson C, Needham D, Haines K, Bailey M, Ward A, Harrold M, et al. Feasibility and inter-rater reliability of the ICU Mobility Scale. Hear Lung J Acute Crit Care 2014;43:19-24.

http://dx.doi.org/10.1016/j.hrtlng.2013.11.003 